\title{
ON ISOMORPHISMS BETWEEN IDEALS IN RINGS OF CONTINUOUS FUNCTIONS $\left({ }^{1}\right)$
}

\author{
BY \\ DAVID RUDD
}

\begin{abstract}
A ring of continuous functions is a ring of the form $C(X)$, the ring of all continuous real-valued functions on a completely regular Hausdorff space $X$.

For an arbitrary ideal $I$ in $C(X)$, the author shows that the maximal ideals of $I$ are precisely the ideals of the form $I \cap M$, for some maximal ideal $M$ in $C(X)$ not containing $I$.

The author shows that any ring isomorphism between ideals in any two rings of continuous functions preserves order, boundedness, and lattice structure; and he uses these results to obtain one of the main theorems: An isomorphism of a maximal ideal in $C(X)$ onto a maximal idea in $C(Y)$ can be extended to an isomorphism of $C(X)$ onto $C(Y)$.

Another of the main theorems characterizes those isomorphisms between $C^{*}(X)$ and $C^{*}(Y)$ (the subrings of bounded functions in $C(X)$ and $C(Y)$ respectively) which can be extended to isomorphisms between $C(X)$ and $C(Y)$.

The author proves that, given any ideal $I$ in $C(X)$, there exists a space $X(I)$ so that the uniform closure of $I$ is isomorphic to a maximal ideal in $C(X(I))$.
\end{abstract}

1. Introduction. Throughout this paper $X$ will denote an arbitrary completely regular Hausdorff space, and $C(X)$ will denote the ring of all real-valued continuous functions on $X$. By a ring of continuous functions is meant a ring of the form $C(X)$ for some space $X$ as above.

Much of the work in rings of continuous functions has been concerned with the ideals of $C(X)$, especially the prime ideals and the maximal ideals. The purpose of this paper is to regard the ideals of $C(X)$ in a somewhat different light, namely as rings in their own right. In studying ideals as rings it quickly becomes apparent that some of the methods used in dealing with all of $C(X)$ are no longer applicable. For example, since $C(X)$ has unity, it is trivial to show that its maximal ideals are prime, but the corresponding result for a maximal ideal of an ideal of $C(X)$ requires some effort. As another example, the existence of square roots of nonnegative functions easily implies that any isomorphism between rings of continuous functions is order preserving. The proof that every isomorphism between ideals of rings of continuous functions is order preserving, however, again requires more work.

Received by the editors July 29, 1970.

AMS 1969 subject classifications. Primary 4625; Secondary 4655.

Key words and phrases. Real-valued continuous functions, structure spaces, extending isomorphisms, $\beta X, v X$.

(1) This paper is based on a part of the author's doctoral dissertation, written under the supervision of Professor James D. McKnight, Jr. The author wishes to thank Professor McKnight for his encouragement and his many helpful suggestions.

Copyright (C) 1971, American Mathematical Society 
In $\S 3$ we discuss the maximal ideals and some structure spaces of an ideal of $C(X)$. We show that given any ideal $I$ of $C(X)$, the maximal ideals of $I$ are precisely those ideals of the form $I \cap M$ where $M$ is a maximal ideal of $C(X)$ not containing I. (This is actually shown true for ideals in rings of a much more general class than rings of continuous functions.) This considerably generalizes a theorem of Kohls $[8$, p. 32].

In $\$ 4$ we discuss isomorphisms between proper ideals of rings of continuous functions. The main lemma is that for any such isomorphism $\varphi, c \cdot \varphi(f)=\varphi(c f)$ for any real number $c$ and any function $f$ in the domain of $\varphi$. This lemma is used to show that $\varphi$ preserves order, boundedness, and lattice structure and that $\varphi$ is continuous in the relative uniform topologies. It is of some interest that this extremely good behavior is enjoyed by any isomorphism between arbitrary proper ideals in any two rings of continuous functions.

In $\$ 5$ we give a procedure for generating new rings of continuous functions from ideals in $C(X)$. We show that, for any ideal $I$ in $C(X)$, there exists a space $X(I)$ and an ideal $J$ of $C(X(I))$, so that $I$ is isomorphic to $J$ and $J$ is contained in a unique (fixed) maximal ideal of $C(X(I))$. We show further that this unique maximal ideal is isomorphic to the uniform closure of $I$; and using this fact and results in $\S 4$ we are able to show that an ideal is uniformly closed if and only if it is isomorphic to a real ideal.

The results of $\S \S 3$ and 4 are applied in $\S 6$ to obtain the main theorem, which states that any maximal ideal of $C(X)$ completely determines $C(X)$ among the rings of continuous functions in the following sense: Any two rings of continuous functions which have isomorphic maximal ideals are themselves isomorphic via an extension of the original isomorphism. Maximality of both of the isomorphic ideals is crucial for this result. An example (6.9) is given of two rings of continuous functions which fail to be isomorphic but have isomorphic ideals, one of which is maximal and the other prime but nonmaximal. Another application is a theorem which provides necessary and sufficient conditions for being able to extend an isomorphism between $C^{*}(X)$ and $C^{*}(Y)$ (these are respectively the subrings of bounded functions in $C(X)$ and $C(Y)$, where $Y$ is of course completely regular and Hausdorff) to an isomorphism between all of $C(X)$ and $C(Y)$.

We wish to point out that Keesling and Nanzetta, working independently from the author, were able to obtain some of the results in this paper. They showed that the maximal ideals of a $z$-ideal $I$ are precisely the ideals of the form $I \cap M$ where $M$ is a maximal ideal in $C(X)$ not containing $I$. They were also able to obtain a partial version of Theorem 6.6.

2. Preliminaries and notations. The letter $R$ will denote the set of all real numbers, and the word "space" will always denote a completely regular Hausdorff topological space.

For any continuous real-valued function $f$, we let $Z(f)$ denote $f^{-1}\{0\}$. 
We use the symbol $\simeq$ to denote homeomorphism between spaces and the symbol $\approx$ to denote isomorphism between rings.

We shall make frequent use of such well-known algebraic results as the following:

(1) The second isomorphism theorem. If $\mathscr{S}$ is a ring and $I$ and $J$ are ideals in $\mathscr{S}$, then $I /(I \cap J) \approx(I+J) / J$.

(2) If $\mathscr{S}$ is a ring and $I$ is an ideal in $\mathscr{S}$ so that $I \cap T=\varnothing$, for some multiplicative semigroup $T$ in $\mathscr{S}$, then there exists a prime ideal $P$ in $\mathscr{S}$ so that $P \supset I$ and $P \cap T=\varnothing$.

The remaining preliminaries are of a somewhat more special nature and are organized into sections.

Most of the material in $\$ 2.1,2.2$, and 2.4 is discussed in [3]. The results in $\S 2.5$ can be found in [6].

2.1. Maximal ideals in $C(X)$. For any maximal ideal $M$ in $C(X)$, either $C(X) / M$ is isomorphic to $R$ or else $C(X) / M$ is a totally ordered nonarchimedean field containing an isomorphic copy of $R$. In the former case we say that $M$ is real, and in the latter, we say that $M$ is hyper-real. If $M$ is real, then for each $f$ in $C(X)$, there exists a unique real number $c$ so that $f-c \in M$. (Of course $c=\xi(f+M)$, where $\xi$ is the unique isomorphism of $C(X) / M$ onto $R$.) We shall identify $c$ with $f+M$. If $M$ is not real, then for each $f$ in $C(X)$, either $|f+M|$ is infinitely large or there exists a unique real number $c$ so that $|(f+M)-c|$ is infinitely small or zero.

We say that a maximal ideal $M$ in $C(X)$ is fixed if, for some $x \in X, M=M_{x}$ $=\{f \in C(X) \mid f(x)=0\}$. Every fixed maximal ideal is real, but the converse is not true in general. A space $X$ is compact if and only if every maximal ideal in $C(X)$ is fixed. A space $X$ is said to be realcompact if every real ideal is fixed.

If $X$ and $Y$ are compact spaces and $\varphi$ is an isomorphism of $C(X)$ onto $C(Y)$, then $\varphi$ induces a one-to-one correspondence between the maximal ideals of $C(X)$ and $C(Y)$. Since these are all fixed maximal ideals, we may define $\tau: X \rightarrow Y$ by $\tau(x)=y$, where $\varphi\left(M_{x}\right)=M_{y}$. The mapping $\tau$ will be a homeomorphism of $X$ onto $Y$. A similar result follows if $X$ and $Y$ are realcompact, since an isomorphism preserves real ideals.

Each maximal ideal $M$ is prime in $C(X)$, and hence absolutely convex, i.e. if $f \in M$ and $g \in C(X)$, then $|g| \leqq|f|$ implies that $g \in M$. (See 5.5 in [3].)

Each maximal ideal $M$ is also a $z$-ideal in $C(X)$, i.e. if $f \in M$, and $Z(g) \supset Z(f)$, then $g \in M$. (See 2.7 in [3].)

2.2. The spaces $v X$ and $\beta X$. For any completely regular Hausdorff space $X$, there exist unique spaces $v X$ and $\beta X$ with the following properties:

(1) $X \subset v X \subset \beta X$.

(2) $X$ is dense in $\beta X$ (hence in $v X$ ).

(3) $C(v X) \approx C(X)$ and $C(\beta X) \approx C^{*}(X)$.

(4) $v X$ is realcompact and $\beta X$ is compact.

(5) Every $f$ in $C(X)$ can be extended uniquely to $f^{v}$ in $C(v X)$. 
(6) Every $f$ in $C^{*}(X)$ can be extended uniquely to $f^{\beta}$ in $C(\beta X)$.

Since $X$ is dense in $\beta X$, no point $p$ in $\beta X \backslash X$ can be isolated. Thus if $p$ is in $\beta X \backslash X$, no neighborhood of $p$ (in $\beta X$ ) can have a finite intersection with $X$.

For a subset $A$ of $\beta X$, we denote the closure of $A$ in $\beta X$ by $A^{\beta}$.

2.3. Structure spaces. If $\mathscr{S}$ is a ring and $\mathscr{P}$ is a collection of prime ideals in $\mathscr{S}$, then $\mathscr{P}$ can be topologized in any of the following equivalent ways:

(1) $\mathscr{A} \subset \mathscr{P}$ is closed if and only if there exists an ideal $I$ in $\mathscr{S}$ so that $\mathscr{A}=\{P \in \mathscr{P} \mid P \supset I\}$.

(2) For $\mathscr{A} \subset \mathscr{P}, \mathrm{cl}(\mathscr{A})=\{P \in \mathscr{P} \mid P \supset \bigcap \mathscr{A}\}$.

(3) For each $s \in \mathscr{S}$, let $E(s)=\{P \in \mathscr{P} \mid s \in P\}$, and let $\mathscr{E}=\{E(s) \mid s \in \mathscr{S}\}$. Then $\mathscr{E}$ is a basis for the closed sets in $\mathscr{P}$, i.e. a subset of $\mathscr{P}$ is closed if and only if it is an intersection of members of $\mathscr{E}$.

The above topology is called the hull-kernel topology (or Stone topology), and we say that $\mathscr{P}$ is a structure space of $\mathscr{S}$. If $\mathscr{P}$ is the collection of all prime maximal ideals of $\mathscr{S}$, then we shall refer to $\mathscr{P}$ as the structure space of $\mathscr{S}$.

We remark that a condition somewhat weaker than primeness is sufficient to ensure that the above definition actually defines a topological space. (See [11].)

The main results which we use about structure spaces are the following:

(1) The structure space $\mathscr{M}$ of $C(X)$, i.e. the space of all maximal ideals of $C(X)$ in the hull-kernel topology, is homeomorphic to the Stone-Čech compactification $\beta X$ of $X$. In the notation of [3], this homeomorphism can be thought of as $M \rightarrow p$, where $M=M^{p}$. (See Exercise $7 \mathrm{~N}$ in [3].)

(2) The space of all real maximal ideals of $C(X)$ is homeomorphic to $v X$; and the extension of $f$ in $C(X)$ to $f^{v}$ is defined by $f^{v}(M)=c$, where $f-c \in M$, for any real maximal ideal $M$. (See [4] for more about these two structure spaces.)

(3) Let $\mathscr{P}$ be a structure space of a ring $\mathscr{S}$, and suppose $K$ is an ideal of $\mathscr{S}$ so that $\cap \mathscr{P} \neq K$. Define $\rho K=\{K \cap P \mid P \in \mathscr{P}$ and $P \neq K\}$. Then $\rho K$ is a collection of prime ideals in $K$ (see Lemma 2 in [10]), and hence admits the hull-kernel topology. Then $\tau: \rho K \rightarrow \mathscr{P}$, defined by $\tau(K \cap P)=P$, is a homeomorphism into $\mathscr{P}$ with open range in $\mathscr{P}$. (See 5.2 in [9].)

2.4. Topologies on $C(X)$. In this paper we will be concerned with two topologies on $C(X)$, the uniform topology and the $m$-topology. The uniform topology is defined by taking a basic neighborhood of a point $f$ in $C(X)$ to be $U_{\varepsilon}(f)$ $=\{g \in C(X)|| f-g \mid<\varepsilon\}$, for any positive real number $\varepsilon$. The $m$-topology is defined similarly; a basic neighborhood of $f$ in $C(X)$ is $U_{\pi}(f)=\{g \in C(X)|| f-g \mid<\pi\}$, for any positive $\pi$ in $C(X)$. (The function $\pi$ is said to be positive, written $\pi>0$, if $\pi(x)>0$ for all $x \in X$.)

Some remarks on these two topologies follow.

(1) The uniform topology. If $A \subset C(X)$, we denote by $A^{u}$ the closure of $A$ in the uniform topology. $C(X)$ is not in general a topological ring in this topology. In particular, there exist ideals whose closures are not ideals. However, for any ideal $I$ in $C(X), I^{u}$ is always a subring of $C(X)$, and it is proper if $I$ is proper. 
D. Plank has shown in [12] that, for a maximal ideal $M$ in $C(X), f \in M^{u}$ if and only if $|f+M|$ is infinitely small or zero. (Actually Plank's result was considerably more general.) From this we can conclude that $M$ is real if and only if $M$ is uniformly closed.

If we let $R^{*}$ denote the one-point compactification of $R$, then for any $f$ in $C(X)$, there exists a unique extension $f^{*}$ of $f$, with $f^{*}: \beta X \rightarrow R^{*}, f^{*}$ continuous of course. Regarding $\beta X$ as the structure space of $C(X), f^{*}$ can be shown to satisfy the following:

If $|f+M|$ is infinitely large, then $f^{*}(M)=\infty$.

If for some $c,|(f+M)-c|$ is infinitely small or zero, then $f^{*}(M)=c$. (See p. 103 in [3].)

Thus $f^{*}(M)=0$ if and only if $|f+M|$ is infinitely small or zero, which is equivalent to $f \in M^{u}$.

If $M$ is a maximal ideal of $C(X)$, then $M^{*}$, defined to be $\left\{f \in C^{*}(X)|| f+M \mid\right.$ is infinitely small or zero\}, is a maximal ideal of $C^{*}(X)$. (See p. 104 in [3].) Furthermore, ${ }^{*}$ is a one-to-one correspondence from the set of maximal ideals of $C(X)$ onto the set of maximal ideals of $C^{*}(X)$. It is evident that $M^{*}=\left(M \cap C^{*}(X)\right)^{u}$ $=M^{u} \cap C^{*}(X)$. If $M$ is hyper-real, then $M^{*}$ contains a unit of $C(X)$, and conversely.

(2) The $m$-topology. If $A \subset C(X)$, we denote by $A^{m}$ the closure of $A$ in the $m$ topology. $C(X)$ is a topological ring in the $m$-topology, and hence every maximal ideal is $m$-closed.

The $m$-topology coincides with the uniform topology if and only if $X$ is pseudocompact, i.e. $C^{*}(X)=C(X)$.

2.5. The ideal $m I$. For any ideal $I$ in $C(X)$, we define $m I=\{f \mid f \in f I\}$. It is easily seen that $m I$ is an ideal of $C(X)$ and that $I \subset J$ implies $m I \subset m J$. We present here a summary of some of the main facts about $m I$ and its relation to the $m$-topology. (See [6].)

(1) $m I=\{f|Z(f) \supset X| Z(h) \supset Z(g)$, for some $h \in C(X)$ and $g \in I\}$.

(2) $m\left(I^{m}\right)=m I$.

(3) $m(m I)=m I$.

(4) $(m I)^{m}=I^{m}$.

(5) $m(I+J)=m I+m J$.

(6) $m I=\bigcap\{m M \mid M \supset I$, and $M$ is a maximal ideal in $C(X)\}$.

In the notation of [3], $f \in m\left(M^{p}\right)$ if and only if there exists $U$ open in $\beta X$ so that $p \in U \subset(Z(f))^{\beta}$. (See p. 106 in [3].)

Using (4) above and the fact that maximal ideals are $m$-closed, it is easily seen that a maximal ideal $M$ contains $I$ if and only if $M$ contains $m I$.

Given any prime ideal $P$ contained in a maximal ideal $M$, it is easily seen that $m M \subset P \subset M$. Taking $m$-closures and using (4), we infer that $P^{m}=M$. Hence a prime ideal is contained in a unique maximal ideal. It can be shown further that for any ideal $I, I$ is contained in a unique maximal ideal $M$ if and only if $I \supset m M$. 
For any ideal $I, m I \subset I \subset I^{m}$ and, if $J$ is an ideal with $m I \subset J \subset I^{m}$, then (2) and (4) above imply that $m I=m J$. Thus the ideals $I^{m}$ and $m I$ serve respectively as upper and lower extremals for separating the ideals of $C(X)$ into equivalence classes. (See [6].)

It is apparent that, for any isomorphism $\varphi$ taking an ideal $I$ onto an ideal $J$, $\varphi(m I)=m J$.

3. Prime and maximal ideals of an ideal. Most of the results of this section are stated and proved for commutative rings, although many are easily generalized to the noncommutative case.

We begin with a purely algebraic result.

3.1. Lemma. Let $\mathscr{S}$ be a ring and $K$ an ideal in $\mathscr{S}$. Then an ideal $A$ of $K$ is prime in $K$ if and only if $A=K \cap P$ for some $P$ prime in $\mathscr{S}$. Furthermore, $P$ is unique if $A$ is proper.

Proof. See [10, Lemma 2] for the sufficiency and 5.1 in [9] for the necessity. The uniqueness follows easily.

3.2. THEOREM. Let $\mathscr{S}$ be a commutative ring and $M$ a maximal ideal in $\mathscr{S}$. Then either (i) $M$ is prime and $\mathscr{S} \mid M$ is a field or (ii) $M$ is not prime and $M \supset \mathscr{S}^{2}$.

Proof. See p. 173 in [5].

3.3. Lemma. Let $\mathscr{S}$ be a commutative algebra over the rationals. Then each maximal ideal of $\mathscr{S}$ is prime.

Proof. Let $M$ be a maximal ideal of $S$. By Theorem 3.2, it suffices to show that $M$ does not contain $\mathscr{S}^{2}$. To this end, assume $M \supseteq \mathscr{S}^{2}$ and form $M^{\prime}=M$ $+\{n x \mid n=0, \pm 1, \pm 2, \ldots\}$, where $x$ is an element in $\mathscr{S} \backslash M$. Clearly $M^{\prime}$ is an ideal of $\mathscr{S}$ which properly contains $M$, whence $M^{\prime}=\mathscr{S}$. Thus, in particular, $\left(\frac{1}{2}\right) x=m+n x$ for some $m \in M$ and some integer $n$. It follows that $2 m=(1-2 n) x$. But $(1-2 n)^{-1} x$ $=m_{1}+n_{1} x$, for some $m_{1} \in M$ and some integer $n_{1}$, whence $x=(1-2 n) m_{1}+2 n_{1} m \in M$, a contradiction.

3.4. THEOREM. Let $\mathscr{S}$ be a commutative algebra over the rationals. Let $K$ be an ideal of $\mathscr{S}$. Then an ideal $D$ of $K$ is a prime maximal ideal in $K$ if and only if $D=M \cap K$ for some maximal ideal $M$ in $\mathscr{S}$, with $M \pm K$. Furthermore, $M$ is unique.

Proof. Let $D=M \cap K$ with $M \nsubseteq K$ and $M$ maximal in $\mathscr{S}$. Then $K / D \approx(M+K) / M$ $=\mathscr{S} \mid M$, which implies that $D$ is a prime maximal ideal of $K$.

Conversely, suppose $D$ is a prime maximal ideal of $K$. By Lemma 3.1, there exists $M$ prime in $\mathscr{S}$ so that $D=K \cap M$. We claim that $M$ is a maximal ideal in $\mathscr{S}$. To see this, assume there exists an ideal $M^{\prime}$ in $\mathscr{S}$ with $M \subset M^{\prime} \subsetneq \mathscr{S}$. Let $s \in \mathscr{S} \backslash M^{\prime}$. The residue class ring $K / D$ is a field, with unity $e+D$ say. Then $e^{2} s+D=e s+D$, which implies that $e(e s-s) \in D$. Since $M$ is prime and $e \notin M$, this implies that es $-s \in M$. Since $D=K \cap M \subset K \cap M^{\prime} \subset K$, either $K \cap M^{\prime}=D$ or $K \cap M^{\prime}=K$. 
But $e s-s \in M^{\prime}$, whence $e s \notin M^{\prime}$, so we must have that $K \cap M^{\prime}=D$. Now let $x \in M^{\prime}$. Then $x e \in D$, which implies that $x e \in M$. Hence $x \in M$, and we have shown that $M^{\prime}=M$.

The uniqueness follows from Lemma 3.1.

We remark that Theorem 3.4 is not true for arbitrary maximal ideals of $K$. Consider the following:

3.5. ExAmple $\left({ }^{2}\right)$. Let $\mathscr{S}$ be the rationals with zero ring multiplication and ordinary multiplication as an algebra over the rationals. Let $K$ be the set of integers. Then $K$ is an ideal in $\mathscr{S}$, and the set of even integers is a maximal ideal in $K$ which cannot be an intersection of $K$ with a maximal ideal of $\mathscr{S}$.

3.6. CoRollary. Let $\mathscr{S}$ be a commutative algebra over the rationals with unity. Let $K$ be an ideal of $\mathscr{S}$. Then an ideal $D$ of $K$ is a maximal ideal of $K$ if and only if $D=M \cap K$ for some maximal ideal $M$ in $\mathscr{S}$, with $M \pm K$.

Proof. Since $\mathscr{S}$ has unity, $K$ is also an algebra over the rationals; hence the maximal ideals of $K$ are prime by Lemma 3.3.

A natural question to ask is which ideals of an ideal $K$ are contained in maximal ideals of $K$. We can answer this question completely for the ideals of $C(X)$.

3.7. THEOREM. Let $I$ be an ideal of $C(X)$. Then an ideal $A$ of $I$ is contained in a maximal ideal of $I$ if and only if $A$ does not contain $m I$.

Proof. Suppose $A \neq m I$. Since $m I=m(m I)$, there exists $f \in I \backslash A$ so that $f=f g$, for some $g \in m I$. Form $G=\left\{g, g^{2}, g^{3}, \ldots\right\}$, a multiplicative semigroup in $I$ which misses $A$. (If $g^{n} \in A$, then $f=f g^{n} \in A$.) Hence there exists $P$ prime in $C(X)$ so that $A \subset I \cap P$ and $P \cap G=\varnothing$. Let $M$ be the maximal ideal in $C(X)$ containing $P$. Then $M \oplus I$, for otherwise, $m I \subset m M \subset P$, which implies that $g \in P$. Thus $A \subset I \cap M$, a maximal ideal in $I$.

Conversely, if $A$ is contained in a maximal ideal of $I$, it must be of the form $I \cap M$, for some $M$ maximal in $C(X)$. Thus if $A \supset m I$, we would have $m I \subset I \cap M \subset M$, whence $M \supset I$.

3.8. Corollary. Let I be an ideal in $C(X)$. If $A$ is prime in I and $A$ does not contain $m I$, then $I|A \approx C(X)| P$, where $P$ is prime in $C(X)$ and $A=I \cap P$.

Proof. Since $A \ngtr m I, A$ is contained in a maximal ideal of $I$, say $I \cap M$, where $M$ is maximal in $C(X)$. Hence $I+M=C(X)$, which implies $m I+m M=C(X)$. But $I \cap P \subset I \cap M$, which implies that $P \subset M$, and hence $m M=m P$ and $m I+m P=C(X)$. Thus $I+P=C(X)$, and $I / A \approx(I+P) / P=C(X) / P$.

We remark that without the condition that $A \ngtr m I$, the result may fail. For example, let $P$ be prime in $C(X)$ with $P \subset M$, a maximal ideal in $C(X)$. Then $M / P$ is clearly not isomorphic to $C(X) / P$, since the latter has unity and the former does not. Of course, $P \supset m M$.

(2) This example was communicated to the author by Professor McKnight. 
3.9. Remark. By Corollary 3.6, if $I$ is an ideal of $C(X)$, then its maximal ideals are precisely the ideals of the form $I \cap M$, where $M$ is maximal in $C(X)$ and $M$ does not contain $I$. Let us denote by $I_{M}$ the intersection of $I$ with $M$, and let $\beta I=\left\{I_{M} \mid M \ngtr I\right.$ and $M$ is maximal in $\left.C(X)\right\}$. Then $\beta I$ is the collection of all maximal ideals of $I$, and it admits the hull-kernel topology. The mapping $\tau: \beta I \rightarrow \mathscr{M}$, defined by $\tau\left(I_{M}\right)=M$ is a homeomorphism into $\mathscr{M}$ (see 2.3). Since $\mathscr{M} \simeq \beta X$, and $\tau(\beta I)$ is open in $\mathscr{M}$ (see 2.3 ), $\beta I$ is locally compact and Hausdorff. The one-point compactification of $\beta I$ is evidently homeomorphic to $(\mathscr{M} \backslash F) \cup\{F\}$, where $F=\{M \in \mathscr{M} \mid M \supseteq I\}$ is shrunk to a point in $\mathscr{M}$. In particular, if $I$ is contained in a unique maximal ideal, then the one-point compactification of $\beta I$ is homeomorphic to $\beta X$.

3.10. Remark. It is evident that a maximal ideal $I_{M}$ in $I$ is real in $I$, i.e. $I / I_{M} \approx R$, if and only if $M$ is real in $C(X) .(I /(I \cap M) \approx(I+M) / M=C(X) / M$. $)$ Thus we may define $v I=\left\{I_{M} \in \beta I \mid M\right.$ is real in $\left.C(X)\right\}$ and the mapping $\tau: v I \rightarrow v X$, defined by $\tau\left(I_{M}\right)=M$, is a homeomorphism into $v X$. In particular, if $I$ is contained in no real maximal ideal of $C(X)$, then $v I \simeq v X$.

4. General properties of isomorphisms. In the following $I$ and $J$ are arbitrary proper ideals of $C(X)$ and $C(Y)$ respectively. We shall denote by $\Delta I$ the set of all $x \in X$ so that $M_{x} \supset I$.

4.1. Lemma. Let $M$ be real in $C(X)$ with $M \neq I$, and let $I_{M}=I \cap M$. Let $e+I_{M}$ be the unity in $I / I_{M}$. Then for any $g \in I, g+I_{M}=c e+I_{M}$ where $g-c \in M$. Conversely, if $g+I_{M}=c e+I_{M}$, then $g-c \in M$.

Proof. Since $M$ is real, there exists $c \in R$ with $g-c \in M$. Thus $g+I_{M}=g e+I_{M}$ and $e(g-c) \in I_{M}$, which implies that $g e+I_{M}=c e+I_{M}$. Conversely, if $g+I_{M}=c e+I_{M}$, then $g e+I_{M}=c e+I_{M}$, which implies that $g-c \in M$.

4.2. THEOREM. Let $\varphi$ be an isomorphism of I onto J. Then $\varphi(c f)=c \varphi(f)$ for all $c \in R$ and all $f \in I$.

Proof. Let $y \in Y$ so that $y \notin \Delta J$ and form $J_{y}=J \cap M_{y}$. ( $M_{y}$ denotes the set of all functions in $C(Y)$ which vanish at $y$.) Then $J_{y}$ is a real maximal ideal of $J$, and hence $J_{y}=\varphi\left(I_{M}\right)$ for some real maximal ideal $M$ in $C(X)$. Let $e+I_{M}$ be the unity in $I / I_{M}$ and denote $\varphi(e)$ by $u$. It is clear that $u+J_{y}$ is the unity in $J / J_{y}$. Let $r \in R$ and consider $r \rightarrow r e+I_{M} \rightarrow \varphi(r e)+J_{y} \rightarrow r^{\prime} u+J_{y} \rightarrow r^{\prime}$ where $\varphi(r e)-r^{\prime} \in M_{y}$. Evidently this defines an isomorphism of $R$ onto $R$; hence the above mapping is the identity. We have shown that $\varphi(r e)+J_{y}=r u+J_{y}$, for $\varphi(r e)-r \in M_{y}$ implies that $u \varphi(r e)-r u \in J_{y}$. Now let $f \in I$ and $c \in R$. By Lemma 4.1, $f+I_{M}=c^{\prime} e+I_{M}$, where $f-c^{\prime} \in M$. Thus we have (1) $\varphi(f)+J_{y}=\varphi\left(c^{\prime} e\right)+J_{y}=c^{\prime} u+J_{y}$. Now $f-c^{\prime} \in M$, so $e c f-e c c^{\prime} \in I_{M}$, whence $\varphi\left(e c c^{\prime}\right)+J_{y}=\varphi(e c f)+J_{y}$. But this implies that $u c c^{\prime}+J_{y}$ $=\varphi(c f)+J_{y}$. Multiplying equation (1) by $u c+J_{y}$, we obtain $u c \varphi(f)+J_{y}=u c c^{\prime} u+J_{y}$, which implies that $c \varphi(f)+J_{y}=u c c^{\prime}+J_{y}$, and hence $c \varphi(f)+J_{y}=\varphi(c f)+J_{y}$. Thus for 
any $y \notin \Delta J, c \varphi(f)-\varphi(c f) \in J_{y}$, which implies that the functions $c \varphi(f)$ and $\varphi(c f)$ have the same value at $y$. Since $c \varphi(f)$ and $\varphi(c f)$ agree at any $y \in \Delta J$, (they are both zero) the theorem is proved.

We remark that Theorem 4.2 is not true in general for ring isomorphisms between commutative algebras over the reals. For example, there exist automorphisms of the complex numbers which do not leave the real numbers fixed. (See [7].)

The above theorem leads to a very useful extension of the isomorphism $\varphi$. We define $(I)=\{f+c \mid f \in I$ and $c \in R\}$ and $(J)$ similarly. Observe that the representation in $(I)$ (and in $(J)$ ) is unique, for if $f_{1}+c_{1}=f_{2}+c_{2}$, then $f_{1}-f_{2}=c_{2}-c_{1} \in I$, whence $c_{2}-c_{1}=0$ and $f_{1}-f_{2}=0$. It is evident that $(I)$ and $(J)$ are subrings.

4.3. Theorem. Let $\varphi$ be an isomorphism of $I$ onto $J$. Then $(I)$ and $(J)$ are isomorphic via an extension of $\varphi$.

Proof. Define $\Phi(f+c)=\varphi(f)+c$, a mapping from $(I)$ into $(J)$. Then $\Phi\left(f_{1}+c_{1}+f_{2}+c_{2}\right)=\varphi\left(f_{1}+f_{2}\right)+c_{1}+c_{2}$ which equals $\Phi\left(f_{1}+c_{1}\right)+\Phi\left(f_{2}+c_{2}\right)$. Also $\Phi\left[\left(f_{1}+c_{1}\right) \cdot\left(f_{2}+c_{2}\right)\right]=\varphi\left(f_{1} c_{2}+f_{2} c_{1}+f_{1} f_{2}\right)+c_{1} c_{2}$ which equals $\varphi\left(f_{1} c_{2}\right)+\varphi\left(f_{2} c_{1}\right)$ $+\varphi\left(f_{1}\right) \varphi\left(f_{2}\right)+c_{1} c_{2}$. Using Theorem 4.2, this is $c_{2} \varphi\left(f_{1}\right)+c_{1} \varphi\left(f_{2}\right)+\varphi\left(f_{1}\right) \varphi\left(f_{2}\right)+c_{1} c_{2}$ which equals $\left[\Phi\left(f_{1}+c_{1}\right)\right] \cdot\left[\Phi\left(f_{2}+c_{2}\right)\right]$. Hence $\Phi$ is a homomorphism of the ring $(I)$ into the ring $(J)$. To see that $\Phi$ is one-to-one, suppose $\Phi(f+c)=0$. Then $\varphi(f)+c$ $=0$, whence $\varphi(f)=-c \in J$. Thus $c=0$, and $f=0$. It is obvious that $\Phi$ is onto $(J)$ and that it extends $\varphi$.

4.4. TheOREM. Let $\varphi$ be an isomorphism of I onto J, and let $\Phi$ be the induced isomorphism of $(I)$ onto $(J)$. Then both $\varphi$ and $\Phi$ are order preserving.

Proof. We first establish that $\varphi$ is order preserving. To see this, let $f$ be a nonnegative function in $I$ and let $g$ denote $\varphi(f)$. It suffices to show that $g(y) \geqq 0$ for any $y \in Y \backslash \Delta J$. So consider any such $y$ and form $J_{y}=J \cap M_{y}$. Thus $J_{y}$ is a real ideal in $J$, which implies that there exists $M$ real in $C(X)$ so that $J_{y}=\varphi\left(I_{M}\right)$. Let $e+I_{M}$ be the identity in $I / I_{M}$, and let $u=\varphi(e)$. There exists $c \in R$ with $f-c \in M$, and, since $f$ is nonnegative, we must have $c \geqq 0$. Thus $f+I_{M}=c e+I_{M}$, whence $g+J_{y}=c u+J_{y}$ and $g(y)=c$.

To see that $\Phi$ is order preserving, let $f+c \in(I)$ with $f+c \geqq 0$. Clearly $c \geqq 0$, and if $c=0$, then $\Phi(f+c)=\varphi(f)$ which is nonnegative. Thus we may assume that $c>0$ and form $f^{\prime}=f / \sqrt{ }(f+c+\sqrt{ } c)$. Then $f^{\prime} \in I$ and $f^{\prime}+\sqrt{ } c=\sqrt{ }(f+c)$, which implies that $\sqrt{ }(f+c) \in(I)$. Thus $\Phi(f+c)=\Phi(\sqrt{ }(f+c)) \Phi(\sqrt{ }(f+c)) \geqq 0$.

4.5. CoROllary. Let $\varphi$ be an isomorphism of $I$ onto J. Then $\varphi\left[I \cap C^{*}(X)\right]$ $=J \cap C^{*}(Y)$. In fact, if $|f| \leqq c$, then $|\varphi(f)| \leqq c$, for any $f$ in $I$.

Proof. Let $f \in I$ and suppose for some $c,|f| \leqq c$. Then $f+c \geqq 0$, and hence $\Phi(f+c)=\varphi(f)+c \geqq 0$, since $\Phi$ is order preserving. But this implies that $\varphi(f) \geqq-c$. Similarly, $\varphi(f) \leqq c$. 
4.6. COROLLARY. If $\varphi$ is an isomorphism of I onto $J$, then $\varphi$ is continuous in the relative uniform topologies on $I$ and $J$.

Proof. Since $I$ and $J$ are first countable spaces, it suffices to show that if $f \in I$ and $\left\langle f_{n}\right\rangle$ is a sequence in $I$ converging to $f$, then $\left\langle\varphi\left(f_{n}\right)\right\rangle$ converges to $\varphi(f)$. Now $f_{n} \rightarrow f$ implies that, for any $\varepsilon>0$, there exists an integer $k$, so that for all $n>k$, $-\varepsilon<f_{n}-f<\varepsilon$. Applying $\varphi$ and using Corollary 4.5, we obtain $-\varepsilon<\varphi\left(f_{n}\right)-\varphi(f)<\varepsilon$ for all $n>k$, whence $\varphi\left(f_{n}\right) \rightarrow \varphi(f)$.

We remark that an isomorphism need not be continuous in the relative $m$ topologies. An example will be furnished in $§ 6$.

The next theorem shows that every isomorphism between $I$ and $J$ is, in a sense, a lattice isomorphism. By this we mean that the isomorphism preserves whatever lattice structure $I$ and $J$ possess.

4.7. THEOREM. Let $\varphi$ be an isomorphism of I onto J. Then if $f, g$, and $f \vee g$ are elements of $I, \varphi(f \vee g)=\varphi(f) \vee \varphi(g)$. Similarly for $f \wedge g$.

Proof. It suffices to show that $\varphi(f \vee g)=\varphi(f) \vee \varphi(g)$ at any $y \notin \Delta J$. So consider any such $y$ and form $J_{y}=J \cap M_{y}$. As before, there exists $M$ real in $C(X)$ so that $J_{y}=\varphi\left(I_{M}\right)$. There exist $c_{1}$ and $c_{2}$ in $R$ and $m_{1}$ and $m_{2}$ in $M$ so that $f-c_{1}=m_{1}$ and $g-c_{2}=m_{2}$. We claim $f \vee g-c_{1} \vee c_{2} \in M$, for if not, then for some $a$ in $C(X)$ and $m$ in $M, a\left(f \vee g-c_{1} \vee c_{2}\right)+m=1$. Choose $x$ in $Z\left(m_{1}\right) \cap Z\left(m_{2}\right) \cap Z(m)$. Then $a(x) \cdot\left[(f \vee g)(x)-c_{1} \vee c_{2}\right]=1$. But $(f \vee g)(x)=c_{1} \vee c_{2}$, a contradiction. Now $f+I_{M}$ $=c_{1} e+I_{M}$ implies that $\varphi(f)+J_{y}=c_{1} u+J_{y}$, where $e+I_{M}$ is the unity in $I / I_{M}$ and $u=\varphi(e)$. Since $u-1 \in J_{y}$, this implies that $(\varphi(f))(y)=c_{1}$. Similarly $(\varphi(g))(y)=c_{2}$ and $(\varphi(f \vee g))(y)=c_{1} \vee c_{2}$, which establishes that $\varphi(f \vee g)=\varphi(f) \vee \varphi(g)$. The proof for $\varphi(f \wedge g)$ is similar.

We now introduce another useful extension of an isomorphism.

4.8. THEOREM. Let $\varphi$ be an isomorphism of I onto J. Then $I^{u}$ and $J^{u}$ are isomorphic via an extension of $\varphi$.

Proof. Let $f \in I^{u}$. Then there exists a sequence $\left\langle f_{n}\right\rangle$ in $I$ so that $f_{n} \rightarrow f$. Obviously $\left\langle f_{n}\right\rangle$ is a Cauchy sequence, i.e., given $\varepsilon>0$, there exists an integer $k$ so that $\left|f_{n}-f_{m}\right|<\varepsilon$, for all $n, m>k$. Let $g_{n}$ denote $\varphi\left(f_{n}\right)$. By Corollary $4.5,\left|f_{n}-f_{m}\right|<\varepsilon$ implies that $\left|g_{n}-g_{m}\right|<\varepsilon$, whence $\left\langle g_{n}\right\rangle$ is also a Cauchy sequence. Let $g$ be defined by $g(y)=\lim g_{n}(y)$. Then $g$ is a uniform limit of continuous functions, so $g \in C(Y)$. Clearly $g \in J^{u}$. We shall define $\psi(f)=g$. To see that $\psi$ is well defined, assume that $f_{n}^{\prime} \rightarrow f$ with $f_{n}^{\prime} \in I$. Let $g_{n}^{\prime}=\varphi\left(f_{n}^{\prime}\right)$, and suppose $g_{n}^{\prime} \rightarrow g^{\prime}$. We claim that $g^{\prime}=g$. To see this, let $y \in Y$, and let $\varepsilon>0$ be given. Then there exists $k$ so that for all $n>k$, $-\varepsilon / 3<f_{n}-f_{n}^{\prime}<\varepsilon / 3$, which implies that, for all $n>k,\left|g_{n}-g_{n}^{\prime}\right|<\varepsilon / 3$. Similarly there exists $j$ so that for all $m>j,\left|g(y)-g_{m}(y)\right|<\varepsilon / 3$, and $\left|g^{\prime}(y)-g_{m}(y)\right|<\varepsilon / 3$. Thus there exists an integer $p$ so that, for all $n>p$,

$$
\begin{aligned}
\left|g(y)-g^{\prime}(y)\right| & =\left|g(y)-g_{n}(y)+g_{n}(y)+g_{n}^{\prime}(y)-g_{n}^{\prime}(y)-g^{\prime}(y)\right| \\
& \leqq\left|g(y)-g_{n}(y)\right|+\left|g^{\prime}(y)-g_{n}^{\prime}(y)\right|+\left|g_{n}(y)-g_{n}^{\prime}(y)\right|<\varepsilon .
\end{aligned}
$$


Thus $g=g^{\prime}$. It is evident that $\psi$ is onto $J^{u}$ and that it extends $\varphi$. (For if $f \in I$, choose $f_{n}=f$.) To see that $\psi$ is a homomorphism, consider $\psi\left(f_{1}+f_{2}\right)$. Let $h_{n} \rightarrow f_{1}+f_{2}$ with $h_{n} \in I$, and let $g_{n}=\varphi\left(h_{n}\right)$. Then $g_{n} \rightarrow g \in J^{u}$ and $\psi\left(f_{1}+f_{2}\right)=g$. Now let $a_{n} \rightarrow f_{1}$ and $b_{n} \rightarrow f_{2}$. Then $a_{n}+b_{n} \rightarrow f_{1}+f_{2}$; hence by the above argument, $\varphi\left(a_{n}+b_{n}\right) \rightarrow g$. But $\varphi\left(a_{n}+b_{n}\right)=\varphi\left(a_{n}\right)+\varphi\left(b_{n}\right)$. So if $\varphi\left(a_{n}\right) \rightarrow a \in J^{u}$ and $\varphi\left(b_{n}\right) \rightarrow b \in J^{u}$, we have that $g=a+b$, where $a=\psi\left(f_{1}\right)$ and $b=\psi\left(f_{2}\right)$. Similarly, $\psi\left(f_{1} f_{2}\right)=\psi\left(f_{1}\right)\left(f_{2}\right)$, and we have that $\psi$ is a homomorphism. To see that $\psi$ is one-to-one, suppose $\psi(f)=0$ and $f_{n} \rightarrow f$. Let $g_{n}$ denote $\varphi\left(f_{n}\right)$. Then $g_{n} \rightarrow 0$ in $C(Y)$, which implies that, for any $\varepsilon>0$, there exists $k$ so that $\left|g_{n}\right|<\varepsilon$ for all $n>k$. Applying $\varphi^{-1}$, we obtain $\left|f_{n}\right|<\varepsilon$ for all $n>k$, whence $f_{n} \rightarrow 0$. Thus $f=0$ and $\psi$ is an isomorphism.

4.9. Corollary. Let $M$ and $N$ be maximal ideals in $C(X)$ and $C(Y)$ respectively, and suppose $M$ and $N$ are isomorphic. Then $M^{*}$ and $N^{*}$ are isomorphic.

Proof. By Corollary 4.5, $M \cap C^{*}(X) \approx N \cap C^{*}(Y)$. Since these are ideals in $C^{*}(X)$ and $C^{*}(Y)$ respectively, we may apply Theorem 4.8 to obtain $\left[M \cap C^{*}(X)\right]^{u}$ $\approx\left[N \cap C^{*}(X)\right]^{u}$, which by 2.4 is the required result.

In $\S 6$ we shall give an example (6.9) to show that the converse is not true in general.

The final results of this section concern a natural mapping which is induced by an isomorphism.

4.10. THEOREM. Let $\mathscr{A}$ and $\mathscr{B}$ be commutative rings, and let $\varphi$ be an isomorphism of $\mathscr{A}$ onto $\mathscr{B}$. Suppose that $\mathscr{P}$ and $\mathscr{Q}$ are collections of prime ideals in $\mathscr{A}$ and $\mathscr{B}$ respectively, satisfying the following condition: $Q \in \mathscr{Q}$ if and only if $Q=\varphi(P)$ for some $P \in \mathscr{P}$. Then $\mathscr{P}$ and $\mathscr{Q}$ are homeomorphic in the hull-kernel topology.

Proof. Define $\tau: \mathscr{P} \rightarrow \mathscr{Q}$ by $\tau(P)=\varphi(P)$. By hypothesis, $\tau$ is onto $\mathscr{Q}$ and $\tau$ is one-to-one since $\varphi$ is an isomorphism. To see that $\tau$ is continuous, consider $E(b)$ $=\{Q \in \mathscr{Q} \mid b \in Q\}$, for some $b \in \mathscr{B}$, a basic closed set in $\mathscr{Q}$. Then clearly $\tau^{-1} E(b)$ $=\left\{P \in \mathscr{P} \mid \varphi^{-1}(b) \in P\right\}$, a closed set in $\mathscr{P}$. Similarly, $\tau$ is closed.

\subsection{Corollary. If $I$ and $J$ are isomorphic, then $\beta I \simeq \beta J$ and $v I \simeq v J$.}

Proof. An isomorphism preserves both maximal ideals and real maximal ideals; hence Theorem 4.10 is applicable.

5. The space $X(I)$. We have already used an ideal $I$ to construct two topological spaces, $\beta I$ and $v I$. In this section we will construct a space $X(I)$ whose ring of continuous functions will be intimately related to the ideal $I$ and, in a certain sense, will be constructible from $I$.

So again we let $I$ denote an arbitrary proper ideal in a ring of continuous functions $C(X)$, and we denote by $F$ the set of all maximal ideals in $C(X)$ which contain $I$. Thus $F$ is a closed set in $\beta X$.

With $I$ and $F$ as above, let $X(I)=(X \backslash X \cap F) \cup\{F\}$ topologized as follows: A neighborhood of a point $x \in X \backslash F$ is a neighborhood of $x$ in the relative topology 
of $X \backslash F$. A neighborhood of $F$ is a set of the form $\{F\} \cup[U \cap(X \backslash F)]$, where $U \supset F$ and $U$ is open in $\beta X$. It is easily verified that $X(I)$ is a completely regular Hausdorff space.

We remark that the following are equivalent:

(i) $F$ is open and closed in $\beta X$,

(ii) $\{F\}$ is open in $X(I)$,

(iii) there exists $U$ open in $\beta X$ so that $U \supset F$ and $U \cap(X \backslash F)=\varnothing$.

We shall make use of the uniform closure to obtain $C(X(I))$ from $I$.

5.1. LeMMA. $I^{u}=\left\{h \in C(X) \mid Z\left(h^{*}\right) \supset F\right\}$.

Proof. Clearly if $h \in I^{u}$, then $Z\left(h^{*}\right) \supset F$, for if $M \in F, h \in M^{u}$ which implies that $h^{*}(M)=0$. Now suppose $Z\left(h^{*}\right) \supset F$ and let $\varepsilon>0$ be given. Let

$$
U=\left\{p \in \beta X|| h^{*}(p) \mid<\varepsilon\right\},
$$

an open set in $\beta X$ containing $F$. By the normality of $\beta X$, there exists an open set $V$ and a continuous function $\hat{k}: \beta X \rightarrow[0,1]$ so that $F \subset V \subset V^{\beta} \subset U$ and $\hat{k}(V)=0$, and $\hat{k}(\beta X \backslash U)=1$. We claim that $k=\hat{k} \mid X$ is a member of $m I$. In view of (6) in 2.5 , it suffices to show that $k \in m M$ for any $M \in F$. But this is obvious since we have that $M \in V \subset Z(k)^{\beta}$ for all $M \in F$. Since $k \in m I, k h \in m I$. Consider any $x \in U \cap X$. Then $|h(x)-k(x) h(x)|=|h(x)| \cdot|1-k(x)|<\varepsilon$. On the other hand, if $x \in X \mid U \cap X$, then $k(x)=1$ and $|h(x)-k(x) h(x)|=0$. We have shown that $|h-k h|<\varepsilon$, whence $h \in(m I)^{u} \subset I^{u}$.

5.2. Corollary. $I^{u}=\bigcap\left\{M^{u} \mid M \in F\right\}$.

Proof. Obvious since $h \in M^{u}$ if and only if $h^{*}(M)=0$.

5.3. Corollary. $(m I)^{u}=I^{u}$.

Proof. If $f \in I^{u}$, then $Z\left(h^{*}\right) \supset F$. Proceeding as in the proof of Lemma 5.1, it is easily seen that $f \in(m I)^{u}$.

For each $f \in I$, let $\bar{f}$ be defined as follows: $\bar{f}(x)=f(x)$ if $x \in X \backslash F$, and $\bar{f}(F)=0$. It is easily seen that $\bar{f} \in C(X(I))$; for $f \in I$ implies that $Z\left(f^{*}\right) \supset F$. Thus given $\varepsilon>0$, let $U=\left\{p \in \beta X|| Z\left(f^{*}\right) \mid<\varepsilon\right\}$. Then $\{F\} \cup[U \cap(X \mid F)]$ is a neighborhood of $F$ in $X(I)$ on which $\bar{f}$ has values in $(-\varepsilon, \varepsilon)$.

5.4. THEOREM. Let $\bar{I}=\{\bar{f} \mid f \in I\}, \bar{f}$ as above. Then (1) $\bar{I}$ is isomorphic to $I$ and $\bar{I}$ is an ideal of $C(X(I))$, and (2) $m M_{F} \subset \bar{I} \subset M_{F}$, where $M_{F}=\{h \in C(X(I)) \mid h(F)=0\}$.

Proof. (1) It is clear that $(f+g)^{-}=\bar{f}+\bar{g}$ and that $(f g)^{-}=\bar{f} \cdot \bar{g}$. Now suppose $\bar{f}=0$, and let $x \in X$. If $M_{x} \in F$, then $f \in I \subset M_{x}$, which implies that $f(x)=0$. If $M_{x} \notin F$, then $x \in X \backslash F$, and $\bar{f}(x)=0=f(x)$. We have shown that $\bar{f}=0$ implies $f=0$.

It is clear that $\bar{I}$ is a subring of $C(X(I))$. To see that it is an ideal, let $g \in C(X(I))$ and $\bar{f} \in \bar{I}$. Define $h$ as follows: $h(x)=g(x)$ if $x \in X \backslash F$, and $h(x)=g(F)$ if $x \in X \cap F$. Then $h: X \rightarrow R$ and $h$ is clearly continuous at any point in $X \backslash F$. To see that 
$h \in C(X)$, let $x \in X \cap F$ and let $c$ denote $g(F)$. Then given $\varepsilon>0$, there exists $U$ open in $\beta X$ so that $F \subset U$ and $g$ takes $[U \cap(X \backslash F)] \cup\{F\}$ into $(c-\varepsilon, c+\varepsilon)$. Consider $U \cap X$, a neighborhood of $x$ in $X$, and let $t \in U \cap X$. If $t \in F$, then $h(t)=c$. If $t \notin F$, then $h(t)=g(t) \in(c-\varepsilon, c+\varepsilon)$. Thus $h$ is continuous at $x$ and is a member of $C(X)$. It is evident that $(h f)^{-}=g \bar{f}$ and, since $h f \in I$, this implies that $\bar{I}$ is an ideal in $C(X(I))$.

(2) Let $g \in m M_{F}$. Then there exists $U$ open in $\beta X$ with $F \subset U$ so that $g$ vanishes on $[U \cap(X \backslash F)] \cup\{F\}$. Define $h: X \rightarrow R$ by $h(x)=g(x)$ for $x \in X \backslash F$ and $h(x)=0$ for $x \in X \cap F$. Arguing as in part (1) above, it is easily shown that $h \in C(X)$, and we claim that $h \in I$. (We shall actually show that $h \in m I$.) To see this, let $M \in F$, say $M=M^{p}$ where $p \in \beta X$. For convenience, we regard $p$ as a member of the closed set $F$ in $\beta X$, and we claim that $U \subset Z(h)^{\beta}$. We consider the following two cases.

Case I. $U \cap(X \mid F)=\varnothing$. This implies that $U=F$ and, since $F \cap Z(h)^{\beta} \neq \varnothing$, $U \cap Z(h)^{\beta} \neq \varnothing$, whence $U \subset Z(h)^{\beta}$.

Case II. $U \cap(X \backslash F) \neq \varnothing$. Let $x \in U \cap(X \backslash F)$. Then $h(x)=g(x)=0$, and $U \cap Z(h)^{\beta} \neq \varnothing$.

Thus we have $p \in U \subset Z(h)^{\beta}$, which implies that $h \in m\left(M^{p}\right)$. (See 2.5.) We have shown that $h \in \bigcap\{m M \mid M \in F\}$ which equals $m I$.

We have the following important corollary.

5.5. COROLLARY. Given any proper ideal I in a ring of continuous functions, there exists a space $Y$ and a fixed maximal ideal $M$ in $C(Y)$ so that $m I$ is isomorphic to $m M$.

Proof. Letting $Y=X(I)$ and $M=M_{F}$, we have $I \approx \bar{I}$ and $m M_{F} \subset \bar{I} \subset M_{F}$. This implies that $m I \approx m \bar{I}$, but $m \bar{I}=m M$.

The arguments in the preceding propositions have perhaps made it apparent how one obtains $C(X(I))$ from $I$. Using the notation of $\S 4$, we form the set $\left(I^{u}\right)$ $=\left\{f+c \mid f \in I^{u}\right.$ and $\left.c \in R\right\}$, and we define the mapping $\varphi:\left(I^{u}\right) \rightarrow C(X(I))$ as follows:

$$
\begin{aligned}
& (\varphi(f+c))(x)=f(x)+c \quad \text { for } x \in X \backslash F, \\
& (\varphi(f+c))(F)=c .
\end{aligned}
$$

The verification that $\varphi$ maps into $C(X(I))$ is routine; using the fact that $f \in I^{u}$ implies $f^{*}$ vanishes on $F$, one proceeds as in the discussion preceding Theorem 5.4.

Since $I^{u}$ is proper, no nonzero constant can be a member of $I^{u}$; hence the representation of an element of $I^{u}$ is unique.

5.6. THEOREM. The mapping $\varphi$ defined above is an isomorphism of $\left(I^{u}\right)$ onto $C(X(I))$. Furthermore, $\varphi$ maps $I^{u}$ onto $M_{F}$.

Proof. It is clear that $\varphi$ is a homomorphism. If $\varphi(f+c)=0$, then $f(x)=-c$ for all $x \in X \backslash F$; and $(\varphi(f+c))(F)=0$ implies $c=0$. If $x \in X \cap F$, then $I \subset M_{x}$, which implies that $I^{u} \subset M_{x}$, hence $f \in M_{x}$. We have shown that $c=0$ and $f(x)=0$ for all $x \in X$, whence $f+c=0$. 
To see that $\varphi$ is onto $C(X(I))$, let $g \in C(X(I))$ and let $c$ denote $g(F)$. Define $h: X \rightarrow R$ by $h(x)=g(x)$ for $x \in X \backslash F$, and $h(x)=c$ for $x \in X \cap F$. Arguing as in Theorem 5.4 part (1), $h \in C(X)$. Let $f=h-c$. We claim that $f \in I^{u}$. To see this, let $M \in F$, and suppose $f^{*}(M) \neq 0$, say $f^{*}(M)=\varepsilon>0$. Then there exists a neighborhood $U$ of $M$ in $\beta X$ so that $f^{*}$ is greater than $\varepsilon / 2$ on $U$. The continuity of $g$ implies that there exists $V$ open in $\beta X$ with $F \subset V$ and $g(V \cap(X \backslash F)) \subset(c-\varepsilon / 2, c+\varepsilon / 2)$. Since $U \cap V$ is nonempty $(M \in U \cap V)$, there exists $x \in U \cap V \cap X$. If $x \in F$, then $f^{*}(x)=f(x)=h(x)-c=0$, a contradiction since $f^{*}(x)>\varepsilon / 2$. If $x \notin F, f^{*}(x)=f(x)$ $=h(x)-c=g(x)-c>\varepsilon / 2$, which contradicts the fact that $g(x)<c+\varepsilon / 2$. We have shown that $f^{*}(M)=0$ for all $M \in F$, whence $f \in I^{u}$ by Lemma 5.1. It is evident that $\varphi(f+c)=g$, so we have that $\varphi$ is onto $C(X(I))$.

Since $I^{u}$ is clearly a maximal ideal in $\left(I^{u}\right)$, its image under $\varphi$ is a maximal ideal in $C(X(I))$. But $\varphi\left(I^{u}\right) \subset M_{F}$, which implies that $\varphi\left(I^{u}\right)=M_{F}$.

As a corollary to the above theorem and to 4.8 we obtain

5.7. THEOREM. A proper ideal I is uniformly closed if and only if I is isomorphic to a real maximal ideal in a ring of continuous functions.

Proof. If $I \approx M$ where $M$ is real, then by Theorem $4.8, I^{u} \approx M^{u}$, via an extension of the original isomorphism. But $M$ real implies that $M=M^{u}$, whence $I=I^{u}$.

Conversely, if $I=I^{u}$, then $I \approx M_{F}$ in $C(X(I))$ by Theorem 5.6.

6. Applications. In the first part of this section, $\mathscr{S}$ will denote a commutative algebra over the reals with unity 1 . Our goal will be to develop an extension theorem similar to Theorem 4.3, and the procedure will be almost the same.

6.1. Lemma. Let $K$ be an ideal in $\mathscr{S}$, and let $P$ be a real maximal ideal in $\mathscr{S}$ not containing $K$. Let $\alpha$ denote the isomorphism of $\mathscr{S} \mid K$ onto $R$, and let $K_{P}$ denote $K \cap P$. Then

(1) $\alpha(r+P)=r$ for any $r \in R$,

(2) there exists $u \in K \backslash P$ so that $u-1 \in P$,

(3) for each $f \in K, f+K_{P}=r u+K_{P}$ where $u-1 \in P$ and $r=\alpha(f+P)$.

Proof. (1) Consider $\theta: R \rightarrow R$ defined by $\theta(r)=\alpha(r+P)$. Then $\theta$ is a nonzero homomorphism, and hence the identity.

(2) $K / K_{P} \approx(K+P) / P=\mathscr{S} \mid P \approx R$. Thus $K / K_{P}$ possesses unity, say $u+K_{P}$. Then $\left(u+K_{P}\right)^{2}=u+K_{P}$, whence $u^{2}-u=u(u-1) \in P$. Since $P$ is prime and $u \notin P$, we have that $u-1 \in P$.

(3) Let $f \in K$ and let $r=\alpha(f+P)$. By (1), $r=\alpha(r+P)$, whence $f+P=r+P$ and $f-r \in P$. Let $u$ be as in (2). Then $u f-u r \in K_{P}=f+K_{P}$, so we obtain $f+K_{P}=u f+K_{P}$ $=u r+K_{P}$.

.We now suppose that $\mathscr{S}$ contains an ideal $K$ which is isomorphic to an ideal $I$ of $C(X)$, say $I \approx K$ via $\varphi$. Let $D$ be a maximal ideal in $K$. Then $D=\varphi\left(I_{M}\right)$ for some $M$ maximal in $C(X)$. Clearly $K / D \approx I / I_{M} \approx(I+M) / M=C(X) / M$. Thus $D$ is real in $K$ if and only if $M$ is real in $C(X)$. 
6.2. TheOREM. Let $K$ be an ideal in $\mathscr{S}$ and $I$ an ideal in $C(X)$, and suppose $\varphi$ is an isomorphism of I onto $K$. Then $\varphi(r f)=r \varphi(f)$ for all $r \in R$ and $f \in I$.

Proof. Let $f \in I$ and $r \in R$ and consider any $I_{M}$ real in $I$. Let $D$ denote $\varphi\left(I_{M}\right)$, and let $e+I_{M}$ denote the unity in $I / I_{M}$. By Theorem 3.4, $D=K \cap P$ for some $P$ maximal in $\mathscr{S}$. Since $R \approx K / D \approx(K+P) / P=\mathscr{S} \mid P$, it follows that $P$ is real in $\mathscr{S}$. Let $u=\varphi(e)$. Then evidently $u+D$ is the unity in $K / D$ and $u-1 \in P$. We consider the mapping of $R$ into $R$ defined by $c \rightarrow c e+I_{M} \rightarrow \varphi(c e)+D \rightarrow c^{\prime}$, where $c^{\prime} u+D=\varphi(c e)+D$. (By (3) in Lemma 6.1, such a $c^{\prime}$ exists.) Since the above map is clearly a nonzero homomorphism, $c=c^{\prime}$ and $\varphi(c e)+D=c u+D$.

Now by Lemma $4.1, f+I_{M}=c e+I_{M}$, where $f-c \in M$. Applying $\varphi$, we obtain $\varphi(f)+D=\varphi(c e)+D=c u+D$. Also $f-c \in M$ implies erf-erc $\in I_{M}$, whence $\varphi($ erf $)+D$ $=\varphi(e r c)+D$ and $\varphi(r f)+D=u r c+D$. But $\varphi(f)+D=c u+D$ implies $u r \varphi(f)+D$ $=u r c u+D=u r c+D$. Since $u r \varphi(f)+D=r \varphi(f)+D$, we have that $r \varphi(f)+D=\varphi(r f)$ $+D$. Now $r \varphi(f) \in K$, so there exists $f^{\prime} \in I$ with $r \varphi(f)=\varphi\left(f^{\prime}\right)$. We have shown that for any real ideal $M$ not containing $I, r \varphi(f)-\varphi(r f)=\varphi\left(f^{\prime}\right)-\varphi(r f) \in \varphi\left(I_{M}\right)$, whence $f^{\prime}-r f \in M$. Since $f^{\prime}-r f$ is a member of any real ideal containing $I$, we have that $f^{\prime}-r f \in \bigcap\{M \mid M$ is real in $C(X)\}$, which implies that $f^{\prime}=r f$ and thus $\varphi(r f)=r \varphi(f)$.

We now define, as in $\S 4,(K)=\{k+c \mid k \in K$ and $c \in R\}$ for any ideal $K$ in $\mathscr{S}$. It is clear that $(K)$ is a subring of $\mathscr{S}$.

6.3. TheOREM. Let $K$ be a proper ideal in $\mathscr{S}$ and $I$ an ideal in $C(X)$, and suppose there exists an isomorphism $\varphi$ of $I$ onto $K$. Then $(I)$ and $(K)$ are isomorphic via an extension of $\varphi$.

Proof. Define $\Phi:(I) \rightarrow(K)$ by $\Phi(f+r)=\varphi(f)+r$. The argument is then the same as in Theorem 4.3.

6.4. Corollary. Let $K$ be a proper ideal in $\mathscr{S}$ and $M$ a real ideal in $C(X)$, and suppose there exists an isomorphism $\varphi$ of $M$ onto $K$. Then $\mathscr{S}$ contains a subring $\mathscr{S}^{\prime}$ which is isomorphic to $C(X)$ via an extension of $\varphi$, and $K$ is a real ideal of $\mathscr{S}^{\prime}$.

Proof. By Theorem 6.3, $(M) \approx(K)$ via an isomorphism which extends $\varphi$. Letting $\mathscr{S}^{\prime}=(K)$, the corollary follows since $(M)=C(X)$.

We remark that under the hypotheses of Corollary 6.4 , the extension $\Phi$ may not be onto $\mathscr{S}$, even if $K$ is maximal in $\mathscr{S}$. In fact, $\mathscr{S}$ may not be isomorphic to any ring of continuous functions. Consider the following example.

6.5. ExAmple. Let $X$ be any space with an isolated point $x$, and form $\mathscr{S}=M_{x} \times C$, where $C$ is the set of complex numbers. Then $\mathscr{S}$ has unity (since $M_{x}$ does) and is a commutative algebra over $R$. The set $K=\left\{(f, 0) \mid f \in M_{x}\right\}$ is a maximal ideal of $\mathscr{S}$ which is clearly isomorphic to $M_{x}$. The extension defined in Theorem 6.3 takes $C(X)$ onto $M_{x} \times R$, a proper subring of $\mathscr{S}$; and it is evident that $\mathscr{S}$ is not isomorphic to any ring of continuous functions.

The remaining applications will be concerned with rings of continuous functions. We are now ready to present one of the main theorems. 
6.6. TheOREM. Let $M$ be a maximal ideal in $C(X)$ and $N$ a maximal ideal in $C(Y)$, and suppose $\varphi$ is an isomorphism of $M$ onto $N$. Then $\varphi$ can be extended to an isomorphism of $C(X)$ onto $C(Y)$.

Proof. Case I. Suppose $M$ and $N$ are both real. Then $(M)=C(X)$ and $(N)$ $=C(Y)$, and the extension $\Phi$ in 4.3 is the required isomorphism.

Case II. Suppose $M$ and $N$ are both hyper-real. By Theorem 4.10, $v M \simeq v N$. But $v M \simeq v X$ and $v N \simeq v Y$ by Remark 3.10; so a homeomorphism $\tau: v X \rightarrow v Y$ is induced, where $\tau$ is defined as follows: If $P \in v X$ (i.e. $P$ is a real ideal in $C(X)$ ), then $\varphi(P \cap M)=N \cap Q$ for some $Q \in v Y$. We define $\tau(P)=Q$. The mapping $\tau$ is a homeomorphism, since it is a composition of homeomorphisms discussed in 4.10 and 2.3. Thus $\tau$ induces a natural isomorphism $\alpha$ of $C(X)$ onto $C(Y)$, defined by $\alpha(f)=\left(f^{\nu} \circ \tau^{-1}\right)$ restricted to $Y$. To see that $\alpha$ extends $\varphi$, let $f \in M$. It suffices to show that $(\alpha(f))^{v}=(\varphi(f))^{v}$. To this end, let $Q \in v Y$. Then for some $P \in v X, Q=\tau(P)$, i.e. $\varphi(P \cap M)=N \cap Q$, and $(\alpha(f))^{\nu}(Q)=\left(f^{\nu} \circ \tau^{-1}\right)(Q)$, which equals $f^{\nu}(P)=c$, where $f-c \in P$. Let $c^{\prime}=(\varphi(f))^{\circ}(Q)$. Then $\varphi(f)-c^{\prime} \in Q$. Now $f(f-c) \in M \cap P$, whence $\varphi[f(f-c)]=\Phi[f(f-c)]=\Phi(f) \Phi(f-c)=\varphi(f)[\varphi(f)-c] \in N \cap Q$. Since $Q$ is prime, either $\varphi(f) \in Q$ or $\varphi(f)-c \in Q$. If $\varphi(f) \in Q$, then $c^{\prime}=0$, and $f \in M \cap P$, whence $c=0$. If $\varphi(f)-c \in Q$, then, since $\varphi(f)-c^{\prime} \in Q, c=c^{\prime}$. We have shown that $c$ must equal $c^{\prime}$, i.e. that $(\alpha(f))^{\circ}(Q)=(\varphi(f))^{\nu}(Q)$.

To complete the proof, it suffices to show that a real ideal cannot be isomorphic to a hyper-real ideal. But this is evident from the fact that an isomorphism between two ideals can be extended to their uniform closures, and real ideals are uniformly closed while hyper-real ideals are not.

6.7. Corollary. Let I and J be ideals in $C(X)$ and $C(Y)$ respectively, so that $I$ is contained in a unique maximal ideal $M$ and $J$ is contained in a unique maximal ideal $N$. Suppose there exists an isomorphism $\varphi$ of I onto $J$ and that $M$ and $N$ are either both real or else both hyper-real. Then $\varphi$ can be extended to an isomorphism of $C(X)$ onto $C(Y)$ which takes $M$ onto $N$.

Proof. By the uniqueness of $M$ and $N, m M \subset I \subset M$ and $m N \subset J \subset N$. Suppose $M$ and $N$ are both real. Then $(m M)^{u} \subset M^{u}=M$, and $(m M)^{u} \supset(m M)^{m}=M$, whence $(m M)^{u}=M$. Similarly, $(m N)^{u}=N$, and it follows that $I^{u}=M$ and $J^{u}=N$. Thus the extension $\psi$, defined in Theorem 4.8, takes $M$ onto $N$, and hence it can be extended by Theorem 6.6 to an isomorphism of $C(X)$ onto $C(Y)$.

If $M$ and $N$ are both hyper-real, then neither $I$ nor $J$ is contained in a real ideal, whence $v I \simeq v X$ and $v J \simeq v Y$. The same argument as in 6.6 Case II can be used to provide an isomorphism $\alpha$ of $C(X)$ onto $C(Y)$ which extends $\varphi$. Since $\alpha(m M)$ $=\alpha(m I)=\varphi(m I)=m J=m N$, it is easily seen that $\alpha(M)=N$.

In Example 6.9 we shall give an example of isomorphic ideals contained in unique maximal ideals, one of which is real and one hyper-real.

We may regard $C^{*}(X)$ and $C^{*}(Y)$ as rings of continuous functions on the compact spaces $\beta X$ and $\beta Y$ respectively. A natural question to ask is, when can an 
isomorphism of $C^{*}(X)$ onto $C^{*}(Y)$ be extended to an isomorphism of $C(X)$ onto $C(Y)$ ? The next theorem answers this question.

6.8. TheOREM. Let $\varphi$ be an isomorphism of $C^{*}(X)$ onto $C^{*}(Y)$. Then the following are equivalent:

(1) $\varphi$ can be extended to an isomorphism of $C(X)$ onto $C(Y)$.

(2) Both $\varphi$ and $\varphi^{-1}$ preserve strictly positive functions.

(3) The homeomorphism of $\beta X$ onto $\beta Y$ induced by $\varphi$ takes $v X$ onto $v Y$.

Proof. (1) $\Rightarrow(2)$. This is obvious, for if $f \in C^{*}(X)$ with $f>0$, then clearly $\varphi(f) \geqq 0$. But $\varphi$ can be extended to $\alpha$ say, on all of $C(X)$, so $\alpha(1)=\alpha((1 / f)(f))$ $=\alpha(1 / f) \varphi(f)=1$, which implies that $\varphi(f)>0$. Similarly, $\varphi^{-1}$ preserves strictly positive functions.

(2) $\Rightarrow(3)$. Let $\tau$ be the induced homeomorphism, i.e. $\tau\left(M^{*}\right)=\varphi\left(M^{*}\right)$, and suppose $P^{*} \in v X$. (We are here regarding $\beta X$ as the structure space of $C^{*}(X)$. The points in $v X$ are, of course, all maximal ideals $P^{*}$ where $P$ is real in $C(X)$.) Assume $\tau\left(P^{*}\right)=Q^{*}$, where $Q^{*} \notin v Y$. Then $Q^{*}$ contains a unit of $C(Y)$ (see 2.4). Since $\varphi^{-1}$ preserves strictly positive functions, this implies that $P^{*}$ contains a unit in $C(X)$, a contradiction. The argument that $\tau$ takes $v X$ onto $v Y$ is similar.

(3) $\Rightarrow(1)$. Again let $\tau$ denote the induced homeomorphism, and let $\tau^{\prime}$ denote the restriction of $\tau$ to $v X$. Then $\tau^{\prime}$ is a homeomorphism of $v X$ onto $v Y$, and hence it induces an isomorphism $\alpha$ of $C(X)$ onto $C(Y)$, where $\alpha(f)=f^{v} \circ\left(\tau^{\prime}\right)^{-1}$ restricted to $Y$. To see that $\alpha$ extends $\varphi$, let $f \in C^{*}(X)$. It suffices to show that $(\alpha(f))^{v}=(\varphi(f))^{v}$. To this end, let $Q^{*} \in v Y$. Then there exists $P^{*} \in v X$ so that $\tau\left(P^{*}\right)=Q^{*}$, i.e. $\varphi\left(P^{*}\right)$ $=Q^{*}$. Now $(\alpha(f))^{\nu}\left(Q^{*}\right)=f^{v}\left(P^{*}\right)=c$, where $f-c \in P^{*}$. But $f-c \in P^{*}$ implies $\varphi(f)$ $-c \in Q^{*}$ (by applying $\Phi$ ), whence $(\varphi(f))^{\circ}\left(Q^{*}\right)=c$.

We shall now use the space $X(I)$ discussed in $\S 5$ to obtain a simple example showing several things. First, an isomorphism between ideals need not be continuous in the relative $m$-topologies, and second, it is possible for two ideals to be isomorphic and contained in unique maximal ideals, one of which is real and the other hyper-real.

6.9. Example. Let $N$ be the integers and let $p \in \beta N \backslash N$. Consider $N^{\prime}=N \cup\{p\}$ as a subspace of $\beta N$. It is apparent that this is the space $N\left(M^{p}\right)$, in the notation of $\S 5$. We depart somewhat from standard notation and let $M_{p}=\left\{f \in C\left(N^{\prime}\right) \mid f(p)=0\right\}$. Now by Theorem $5.4, M^{p} \approx\left(M^{p}\right)^{-}$and $\left(M^{p}\right)^{-}$is contained in $M_{p}$ uniquely. But of course, $M^{p}$ is hyper-real and $M_{p}$ is real. Thus, we have shown that it is possible for $m I \approx m J$ even though $I$ is real and $J$ is hyper-real, and so in particular, even though $I$ and $J$ are not isomorphic.

We now wish to use the above example to show that isomorphisms need not be continuous in the $m$-topologies. For convenience, let $\varphi$ denote the isomorphism from $M^{p}$ onto $\left(M^{p}\right)^{-}$. We claim that $\varphi$ is not open in the relative $m$-topologies. To see this, let $V=U_{1 / j}(0)=\left\{f \in M^{p}|| f-0 \mid<1 / j\right\}$, where $1 / j$ is the reciprocal function on $N$. Then $V$ is open in the relative $m$-topology on $M^{p}$. Let $W=\varphi(V)$. 
Clearly 0 (in $C\left(N^{\prime}\right)$ ) is a member of $W$, so if $W$ were open, there would exist $\pi^{\prime} \in C\left(N^{\prime}\right), \pi^{\prime}>0$, with $U_{\pi^{\prime}}(0)$, in $\left(M^{p}\right)^{-}$, contained in $W$. In other words $\left\{g \in\left(M^{p}\right)^{-}|| g \mid<\pi^{\prime}\right\} \subset W$. Now $\pi^{\prime}(p)>0$ implies there exists $\varepsilon>0$ and a $\beta$-open neighborhood $A$ of $p$, with $\pi^{\prime}>\varepsilon$ on $\{p\} \cup(A \cap N)$, i.e. $\pi^{\prime}$ is positive on a neighborhood of $p$ in $N^{\prime}$. By the normality of $\beta N$, there exist $\beta$-open sets $B$ and $C$ and a continuous function $\hat{h}: \beta N \rightarrow[0,1]$, so that $p \in B \subset B^{\beta} \subsetneq C \subset C^{\beta} \subsetneq A$, and $\hat{h}$ is 0 on $B^{\beta}$ and $\hat{h}$ is 1 on $\beta N \mid C$. Then $h=\hat{h} \mid N \in M^{p}$ since $p \in Z(h)^{\beta}$.

Let $\pi$ denote $\pi^{\prime} \mid N$, and consider $h \cdot \pi / 2 \in M^{p}$. Clearly $\varphi(h \cdot \pi / 2) \in U_{\pi^{\prime}}(0)$, whence $\varphi(h \cdot \pi / 2) \in W$. But this implies that $h \cdot \pi / 2 \in V$. Now for all $x \in\left(A \mid C^{\beta}\right) \cap N$, $(h \cdot \pi / 2)(x)=\pi(x) / 2>\varepsilon / 2$. But $\left(A \backslash C^{\beta}\right) \cap N$ is an infinite set, so there exists $n \in N$ with $1 / n<\varepsilon / 2$ and $(h \cdot \pi / 2)(n)>\varepsilon / 2$. Since $h \cdot \pi / 2 \in V$, we have $|h(n) \cdot(\pi / 2)(n)|<1 / n$ for all $n \in N$, and thus we have a contradiction which verifies the claim that $\varphi$ is not open.

6.10. Remarks. (1) The ideal $\left(M^{p}\right)^{-}$is prime in $C\left(N^{\prime}\right)$. To see this, suppose $f g \in\left(M^{p}\right)^{-}$, say $f g=\bar{h}$ for some $h \in M^{p}$. Let $f_{1}=f \mid N$ and $g_{1}=g \mid N$. Then $f_{1} g_{1}=h$, which implies that either $f_{1}$ or $g_{1}$ is a member of $M^{p}$. If $f_{1} \in M^{p}$, then $f=f_{1}$ implies $f \in\left(M^{p}\right)^{-}$. Similarly, $g_{1} \in M^{p}$ implies $g \in\left(M^{p}\right)^{-}$.

Thus the Example 6.9 shows that a maximal ideal can be isomorphic to a prime nonmaximal ideal.

(2) Consider the mapping $\psi: C^{*}\left(N^{\prime}\right) \rightarrow C^{*}(N)$ defined by $\psi(f)=f \mid N$. It is easily seen that $\psi$ is an isomorphism of $C^{*}\left(N^{\prime}\right)$ onto $C^{*}(N)$. Clearly $\psi$ preserves strictly positive functions, but $\psi^{-1}$ does not. Of course, $\psi$ cannot be extended to an isomorphism of $C\left(N^{\prime}\right)$ onto $C(N)$. (See Theorem 6.8.) The preceding example motivates us to make the following definition.

6.11. Definition. A space $X$ is said to be $\beta$-fixed, if given any homeomorphism $H$ of $\beta X$ onto itself, $H(X)=X$.

All first countable spaces are $\beta$-fixed, since no point in $\beta X \backslash X$ can have a countable neighborhood basis. (See 9.6 in [3].)

The space $N^{\prime}$, however, even though a countable space (and hence realcompact), is not $\beta$-fixed. To see this, let $\tau: \beta N \rightarrow \beta N$ be a homeomorphism so that $\tau(q)=p$, where $q$ and $p$ are distinct points in $\beta N \backslash N$. Then $N \subset N^{\prime} \subset \beta N$ implies $\beta N^{\prime}=\beta N$, and $\tau^{-1}: \beta N^{\prime} \rightarrow \beta N^{\prime}$ is a homeomorphism which does not take $N^{\prime}$ onto $N^{\prime}$.

However, we do have the following theorem.

6.12. THEOREM. Let $X$ be realcompact. Then the following are equivalent:

(1) $X$ is $\beta$-fixed.

(2) Every automorphism of $C^{*}(X)$ can be extended to an automorphism of $C(X)$.

(3) Every automorphism of $C^{*}(X)$ preserves strictly positive functions.

(4) For all maximal ideals $M$ and $N$ in $C(X), M^{*} \approx N^{*}$ implies that $M \approx N$.

Proof. The equivalence of (1), (2) and (3) follows directly from Theorem 6.8. 
(4) $\Rightarrow$ (1). Let $H: \beta X \rightarrow \beta X$ be a homeomorphism and suppose $H(p)=x$, where $p \in \beta X \backslash X$ and $x \in X$. Then the ideals $\left(M^{p}\right)^{*}$ and $\left(M_{x}\right)^{*}$ are isomorphic, whence $M^{p} \approx M_{x}$, a contradiction, since $M_{x}$ is real and $M^{p}$ is hyper-real.

(2) $\Rightarrow(4)$. Let $\varphi: M^{*} \rightarrow N^{*}$ be an isomorphism. Then $\varphi$ can be extended to an automorphism of $C(\beta X)$ by Theorem 6.6. We may regard $\varphi$ as an automorphism of $C^{*}(X)$, which can then be extended to an automorphism $\alpha$ of $C(X)$. We claim that $\alpha(M)=N$. If not, then $\alpha(M)+N=C(X)$, whence there exist $n \in N \cap C^{*}(X)$ and $m \in M \cap C^{*}(X)$ so that $\alpha(m)+n=1$. But $m \in M^{*}$ implies $\alpha(m)=\varphi(m)$, which is an element of $N^{*}$, and hence $1 \in N^{*}$, a contradiction.

We remark that 6.12 shows that the converse of Corollary 4.9 does not hold in general.

\section{REFERENCES}

1. E. Čech, On bicompact spaces, Ann. of Math. (2) 38 (1937), 823-844.

2. L. Gillman, M. Henriksen and M. Jerison, On a theorem of Gelfand and Kolmogoroff concerning maximal ideals in rings of continuous functions, Proc. Amer. Math. Soc. 5 (1954), 447-455. MR 16, 607.

3. L. Gillman and M. Jerison, Rings of continuous functions, University Series in Higher Math., Van Nostrand, Princeton, N. J., 1960. MR 22 \#6994.

4. E. Hewitt, Rings of real-valued continuous functions. I, Trans. Amer. Math. Soc. 64 (1948), 45-99. MR 10, 126.

5. N. Jacobson, Lectures in abstract algebra. Vol. I: Basic concepts, Van Nostrand, Princeton, N. J., 1951. MR 12, 794.

6. T. R. Jenkins and J. D. McKnight, Jr., Coherence classes of ideals in rings of continuous functions, Nederl. Akad. Wetensch. Proc. Ser. A 65=Indag. Math. 24 (1962), 299-306. MR 25 \#3360.

7. H. Kestelman, Automorphisms of the field of complex numbers, Proc. London Math. Soc. (2) 53 (1951), 1-12. MR 12, 812.

8. C. Kohls, Ideals in rings of continuous functions, Fund. Math. 45 (1957), 28-50. MR 21 $\# 1517$.

9. - The space of prime ideals of a ring, Fund. Math. 45 (1957), 17-27. MR 20 \#7040

10. N. H. McCoy, Prime ideals in general rings, Amer. J. Math. 71 (1949), 823-833. MR 11, 311.

11. J. D. McKnight, On the characterization of rings of functions, Doctoral Thesis, Purdue University, Lafayette, Ind., 1953 (unpublished).

12. D. Plank, Closed l-ideals in a class of lattice-ordered algebras, Illinois J. Math. (to appear).

OLd DOMinion University, NORFOLK, VIRGINIA 23508 